\title{
Dissolution Testing of Orally Disintegrating Tablets
}

\author{
James Klancke \\ Sr. Director, Analytical Development, CIMA LABS INC, Brooklyn Park, MN
}

email correspondence: jim.klancke@cimalabs.com

\begin{abstract}
Orally disintegrating tablets (ODT) are solid dosage forms that disintegrate in the oral cavity leaving an easy-to-swallow residue.The disintegration times are generally less than one minute. For orally disintegrating tablets, taste-masking of bitter or objectional-tasting drug substances is critical.The taste-masking aspect plays a significant role in dissolution method development, specifications, and testing. The USP 2 paddle apparatus is the most suitable and common choice for orally disintegrating tablets. Discriminating, robust dissolution methods are extremely useful for monitoring process optimization and changes during scale-up of taste-masked bulk drug and tablet manufacture.
\end{abstract}

Introduction

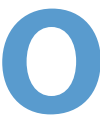
rally disintegrating tablets contain a wide variety of pharmaceutical actives covering many therapeutic categories, and can be particularly good applications for pediatric and geriatric treatments. The time for disintegration of orally disintegrating tablets is generally considered to be less than one minute [1-4], although patients can experience actual oral disintegration times that typically range from 5-30 seconds. Orally disintegrating tablets are characterized by high porosity, low density, and low hardness. When administered, an in-situ suspension is created in the oral cavity as the tablet disintegrates and is subsequently swallowed. Recently, the Orally Disintegrating Tablets terminology has been approved by the Nomenclature and Labeling committee at USP (reference Nomeclature Notes, page 8).

Although fast disintegrating and patient preferred, orally disintegrating tablets are typically not meant to have faster therapeutic onset. In fact, demonstration of bioequivalence is sufficient for product registration, therefore dissolution profiles which match that of a reference listed drug are often sought. Nonetheless, orally disintegrating tablets have gained acceptance and market share, and have achieved reputable status amongst product life cycle management strategies. Patented orally disintegrating tablets technologies include OraSolv ${ }^{\circledR}$, DuraSolv $^{\circledR}$, Zydis $^{\circledR}$, FlashTab ${ }^{\circledR}$, Wowtab ${ }^{\circledR}$, and others. Products representative of these technologies are shown in Table 1.
The development of dissolution methods for orally disintegrating tablets is comparable to the approach taken for conventional tablets, and is practically identical when the orally disintegrating tablet does not utilize taste masking. The reference listed drug may have dissolution conditions in a USP monograph, and is a good place to start with scouting runs for a bioequivalent ODT. Other media such as $0.1 \mathrm{~N}$ hydrochloric acid, and $\mathrm{pH} 4.5$ and 6.8 buffers should be evaluated for orally disintegrating tablets much in the same way as their ordinary tablet counterparts. Experience has shown that the USP 2 paddle apparatus is the most suitable and common choice for orally disintegrating tablets, with a paddle speed of $50 \mathrm{rpm}$ commonly used. Typically, the dissolution of orally disintegrating tablets is very fast when using USP monograph conditions, hence slower paddle speeds may be utilized to obtain a profile.Large tablets

\begin{tabular}{|c|c|}
\hline \multicolumn{2}{|c|}{ Some Commercial Orally Disintegrating Tablet Products } \\
\hline Product & ODTCompany/Partner \\
\hline Alavert ${ }^{\mathrm{TM}}$ Loratadine ODT & CIMA/Wyeth Consumer Health \\
\hline Benadry| ${ }^{\circledR}$ Fastmelt ${ }^{T M}$ & Yamanouchi/Pfizer \\
\hline Claritin $^{\circledR}$ RediTabs ${ }^{\circledR}$ & R.P.Scherer/Schering-Plough \\
\hline Tempra $^{\circledR}$ FirsTabs & CIMA/Mead Johnson \\
\hline Excedrin $^{\circledR}$ QuickTabs $^{\top M}$ & Ethypharm/BMS \\
\hline Maxalt ${ }^{\circledR}$ MLT & R.P.Scherer/Merck \\
\hline NuLev ${ }^{\mathrm{TM}}$ & CIMA/Schwarz Pharma \\
\hline Remeron $^{\circledR}$ SolTabs ${ }^{\mathrm{TM}}$ & CIMA/Organon \\
\hline Triaminic $^{\circledR}$ SoftChews ${ }^{\circledR}$ & CIMA/Novartis Consumer Health \\
\hline Zofran ODT ${ }^{\circledR}$ & R.P.Scherer/Glaxo SmithKline \\
\hline Zomig ZMT®and Rapimelt & CIMA/Astra Zeneca \\
\hline 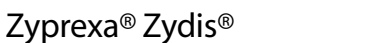 & R.P.Scherer/Eli Lilly \\
\hline
\end{tabular}


approaching or exceeding one gram and containing relatively dense particles may produce a mound in the dissolution vessel, which can be prevented by using higher paddle speeds. These two situations expand the suitable range to 25 - $75 \mathrm{rpm}$. The USP 1 basket apparatus may have certain applications for orally disintegrating tablets, but is used less frequently due to the physical properties of these tablets. Specifically, tablet fragments or disintegrated tablet masses may become trapped on the inside top of the basket at the spindle where little or no effective stirring occurs, yielding irreproducible dissolution profiles.

\section{Taste-Masking Drives Dissolution}

Taste-masking of bitter or objectional-tasting drug substances is critical for any orally-adminstered dosage form, including suspensions and chewable tablets. Less commonly, active pharmaceutical ingredients to be incorporated are tasteless and do not require taste-masking. The taste-masking aspect greatly influences dissolution method development, specifications, and testing. Several factors influence dissolution profiles of orally disintegrating tablets that contain taste-masked actives. Drug particle coatings can vary in thickness, and certain polymer coatings have $\mathrm{pH}$ dependent solubility such as methacrylates which influence dissolution profiles. Surface wettability of these particles is dependent on composition and may also be dependent on the process by which they were manufactured.

There are multiple approaches to taste-masking and coating which have applicability to orally disintegrating tablets and must be understood by method development personnel. A drug solution or suspension can be applied to a substrate followed by polymer coating, or the drug particles may be coated directly. Alternatively, granulation of the drug with certain excipients followed by polymer coating can achieve taste-masking. Certain taste-mask coatings are $\mathrm{pH}$ independent, while others are $\mathrm{pH}$-sensitive. The coatings can dissolve, swell, or become permeable during the dissolution test depending on the selected media. Coating for controlled-release, where taste-masking is a given, can also be used and incorporated into fast-dissolve dosage forms since compression forces are low and the controlled-release particles remain intact. If the drug is tasteless or very low dose, direct blend of bulk drug substance into fast-dissolve matrix is straightforward, and the dissolution profile is influenced primarily by properties of the drug substance.

The disintegration time of orally disintegrating tablets in a dissolution vessel is generally less than thirty seconds and therefore not an important factor in the resulting dissolution profile in terms of discrimination. The discrimination is based on taste-masked coating and the active ingredient itself.Since orally disintegrating tablets are of low density, floating particles may be observed even after complete tablet disintegration has taken place. The disintegration time of an ODT is obvious to patients and product development specialists, so in-vivo and in-vitro times are often compared during the development process. This fast disintegration makes it very important to observe how the tablet fragments and particles are behaving during the dissolution test compared to conventional tablets, which typically display much less breakup at the bottom of the vessel early in the test.

As with all solid oral dosage forms, dissolution serves as a control test. The same is true for taste-masked bulk drug. Batch-to-batch consistency can be assured, and dissolution data on the taste-masked drug is frequently predictive of dissolution of the tableted product. The USP 2 paddle apparatus at 50-100 rpm is suitable for dissolution testing of taste-masked drug as well.The media used for the tastemasked drug should match that of the finished product to maximize the value of the test. There are certain cases where it may be advantageous to use increased paddle speed for taste-masked drug in order to better match the orally disintegrating tablets profile. Dissolution as a stability test for taste-masked drug is indicative of performance of coating over time and is a meaningful test in stability programs. Shelf-life (for bulk warehousing) specifications should match the release specifications.

Dissolution of taste-masked bulk drug is an important test method for both product development and quality control. USP Q-type specifications are not applicable since it is not representative of finished dosage unit (but rather is a homogenous sample).It is appropriate to use the mean value obtained from 6 or 12 vessels. The $\%$ drug released calculation is based directly on assay value of material rather than label claim, and UV analysis of the sample pulls is generally suitable. Filtration is important due to undissolved particles present during sampling. An example of the dependence of dissolution on taste-mask coating thickness for bulk, taste-masked pseudoephedrine $\mathrm{HCl}$ particles is shown in Figure 1 (page 8).

Relative assessment of taste using a neutral $\mathrm{pH}$ media is used to establish approximate baseline for early timepoint dissolution value. The delay in drug release only needs to be long enough to pass through the oral cavity, followed by fast and complete release as for any immediate release dosage form. A simulated ideal profile is shown in Figure 2 (page 8).

The assessment is also monitored during scale-up to assure that the taste-masking attributes are maintained.In 


\section{Orally Disintegrating Tablets ... continued}

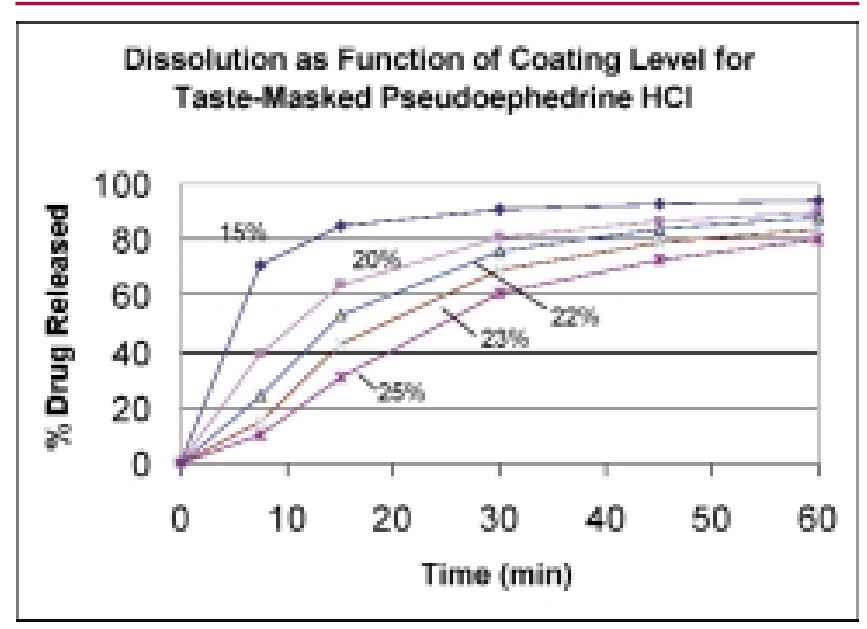

Figure 1.

development, a target specification can be set for tastemasked drug to assure acceptable/consistent taste.

$\mathrm{HPLC}$ is often required to analyze dissolution aliquots due to presence of UV-absorbing components, specifically flavors and sweeteners. Excipient to drug ratios may be higher since the formulation is designed to have good taste and mouthfeel, decreasing signal of the drug to background (excipients) in the UV.

In general, the approach to dissolution for orally disintegrating tablets has similarities to conventional tablets. Dissolution is key to orally disintegrating tablets product development, and taste-masking approaches dictate experimental dissolution plan. Due to the nature of these formulations, there is generally an additional level of complexity in development and analysis.

\section{Nomenclature Notes}

Orally Disintegrating Tablets terminology adapted by the Nomenclature and Labeling committee at USP.

[DRUG] Orally Disintegrating Tablets is the general form of nomenclature for tablets that disintegrate rapidly or instantly in the oral cavity. It is the name to be

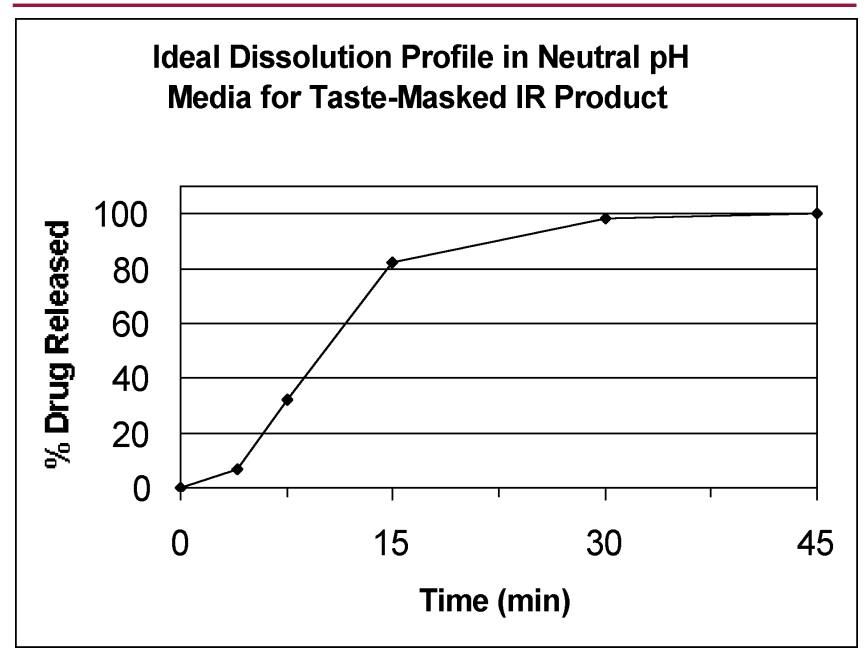

Figure 2.

used where immediate-release is characteristic of the swallowed particles; i.e., drug release is that which is observed also for conventional compressed tablets that are not formulated specifically to release drug at some other time interval, and the Immediate-Release terminology does not appear in the name."

\section{References}

1. AC Liang and LH Chen, Fast-dissolving intraoral drug delivery systems, Expert Opin. Ther. Patents, 11(6), 2001

2. Y Morita, Y Tsusima, M Yasui, R Termoz, J Ajioka, and K Takayama, Evaluation of Disintegration Time of Rapidly Disintegrating Tablets via a Novel Method Utilizing a CCD Camera, Chem. Pharm. Bull., 50(9) 1181-1186, 2002

3. S Schiermeir, and PC Schmidt: Fast dispersible ibuprofen tablets, Eur. J. Pharm Sci. 15 295-305, 2002

4. M Siewert, J Dressman, C Brown, and V Shah, FIP/AAPS Guidelines for Dissolution/In Vitro Release Testing of Novel/Special Dosage Forms, Dissolution Technologies, February 2003 